\title{
Patients' Satisfaction with Day Case Nasal and Sinus Surgery at Manukau Superclinic
}

\author{
Muhammad Shakeel ${ }^{1,2 *}$, Siew Min Keh", Sena Han ${ }^{1}$, Salil Nair ${ }^{1}$ and Zahoor Ahmad \\ ${ }^{1}$ Department of Otolaryngology Head and Neck Surgery, Manukau Superclinics, Auckland, NZ \\ ${ }^{2}$ Department of Otolaryngology-Head and Neck Surgery, Ward 210, Aberdeen Royal Infirmary, Aberdeen, \\ United Kingdom
}

\begin{abstract}
Objective: To evaluate the safety, efficacy, patient satisfaction and feasibility of day case nasal and sinus surgery in our institution.

Method: A retrospective analysis of a prospectively maintained departmental database. In our day stay surgery centre, patients were contacted by telephone on post-op day 1 when they were asked about their overall experiences and any post-operative complications encountered following their surgery. Information was recorded and analyzed using Microsoft Office Excel (2013).

Results: Questionnaires from 160 consecutive patients were analysed. Of 128 patients that were contacted, 113 were available and interviewed. Forty-two children ( 27 males; mean age 10 years) and 71 adults (38 females; mean age 71). Septoplasty, inferior turbinate reduction and sinus surgery were the most common operation performed in adult; turbinate surgery, closed nasal fracture reduction and nasal cautery in children. A 100\% patient satisfaction was reported with day case surgery service. The most common post-op complaint was minimal nasal bleeding (23\%) which settled down with conservative management followed by pain overnight and at interview (VAS $\geq 5,1 \%$ and $2.6 \%$ respectively). There was no delayed discharge or re-admission during the study period.
\end{abstract}

Conclusions: Day stay rhinology surgery, in selected patients, is well-tolerated by both children and adults. The overall satisfaction is high with minimal reported adverse effects.

\section{Keywords}

Day case surgery, Sinonasal surgery, Complication, Patient satisfaction

\section{Introduction}

Day case surgery, which is less than 24-hour hospital stay, has become the norm of ENT surgical practice across the globe and can offer significant advantages to both patients and healthcare providers. Although the major drive to day case has been financial, benefits such as shorter waiting times, less disruption to patients' routine, reduction in nosocomial infection risk can confer significant advantage. When patients are carefully selected, there is no difference in surgical outcomes between day case surgery compared to in-patient surgery [1].

Over the years, there has been a number of publications looking at the feasibility and patient satisfaction undergoing surgery as a day case or an overnight stay. Generally, day surgery is preferred by patients when practical [2-8]. Although certain operations, such as tonsillectomy [2,4-6] and septal surgery, have been well-published, limited published literature exists on patients' satisfaction following the day case sinonasal surgery.

Day case surgery is well accepted for tonsillectomy but limited published literature exists on patients' satisfaction following day case nasal surgery. In our centre, most nasal and sinus surgeries are routinely carried out as day case. In this study, we aim to investigate the feasibility, safety, efficacy and patients' acceptability of day case nasal surgery in our institution.

*Corresponding author: Dr. Muhammad Shakeel, Department of Otolaryngology-Head and Neck Surgery, Ward 210, Aberdeen Royal Infirmary, Aberdeen, AB25 2ZN, United Kingdom

Accepted: February 12, 2022

Published online: February 14, 2022

Citation: Shakeel M, Keh SM, Han S, et al. (2022) Patients' Satisfaction with Day Case Nasal and Sinus Surgery at Manukau Superclinic. J Head Neck Surg 5(1):170-175 


\section{Postop Call - Clinical Assessment/Advice}

patient consent for call YES / NO

Patients Contact numbers

(Home)

(Mobile) (Work)

\section{Preferred Contact Person if unable to contact the Patient}

Name

Relationship Phone No:

Patient consents to RN contacting the above named person to discuss surgery and post-op care: YES / NO

\begin{tabular}{|l|l|}
\hline \multicolumn{1}{|c|}{ Date: } & \\
Wound care plan: & $\begin{array}{l}\text { Interpreter required? Yes / No } \\
\text { Language: }\end{array}$ \\
Type of anaesthesia: GA Sedation Arm block Spinal & \\
\hline Relevant medical history & Surgeon \\
\cline { 2 - 3 } & Anaesthetist \\
\hline $\begin{array}{l}\text { Medications (usual) } \\
\text { Other: }\end{array}$ & Primary Nurse \\
\hline Allergies & \\
\hline
\end{tabular}

Are you comfortable? (Circle as appropriate)

Pain Overnight $\quad / 10 \quad$ Pain score: Now $\quad / 10 \quad$ Dizziness: Yes/No

PONV: Yes/No Eating/ Drinking: Yes/No

What pain medication are you taking? Panadol/ NSAID/Tramadol

$\begin{array}{llllllll}\text { Frequency: } & 4 & 6 & 8 & \text { hourly } & \text { BD } & \text { Daily } & \text { Nil }\end{array}$

Are there any specific problems related to your surgical wound?

Bleeding: Yes / No

Swelling: Yes / No

Dressing Intact: On / Off

PV Loss?

Other?

Refer/ Advice Patient to: GP District Nurse ED A\&E Clinic Clinician Informed: Name:

Were you happy with the service received?

Figure 1: Data collection form. 


\section{Method}

A retrospective analysis over a period of 18 months of a prospectively maintained departmental password protected secured database was carried out.

A standardised questionnaire was created for data collection (Figure 1). Information collected include patient demographics, date of admission, outcome, type of surgery, discharge advice and discharge medications.

In our day stay surgery unit, patients were contacted by telephone on post-op day 1 by the recovery nurses; they were asked about their pain score post-op, any complaint or complication experienced following their surgery, and overall satisfaction score. A maximum of three attempts were made to try to reach patients for telephone interview. Patients who are unavailable for an interview are excluded from this study. In the children patient group, information was provided by a parent or carer when the child was unable to do so. Postoperative pain was evaluated using a visual analogue scale (VAS) for adults (1) or the Wong-Baker FACES pain rating scale for children (2); $0=$ no pain and $10=$ worse imaginable pain. Information was recorded and analysed using Microsoft Office Excel (2013).

\section{Results}

A total of 160 consecutive patients of all ages admitted for sinonasal surgery were identified over an 18-month period. Of 128 patients that were contacted, 113 were available and interviewed (Figure 2). The age of patients included ranged from 3-81 years. Forty-two were children (27 males; mean age 10 years, ranging from 3-14 years) and 71 were adults (38 females; mean age 38 years, ranging from 15-81 years).

All patients had general anesthesia for their nasal and or sinus surgery. Inferior turbinoplasty, closed nasal fracture

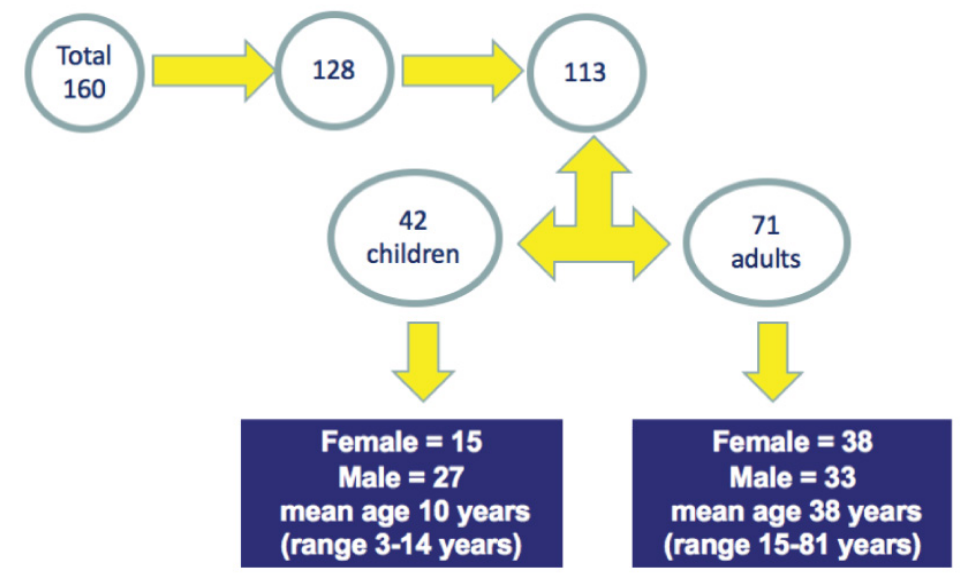

Figure 2: Characteristics of study participants.

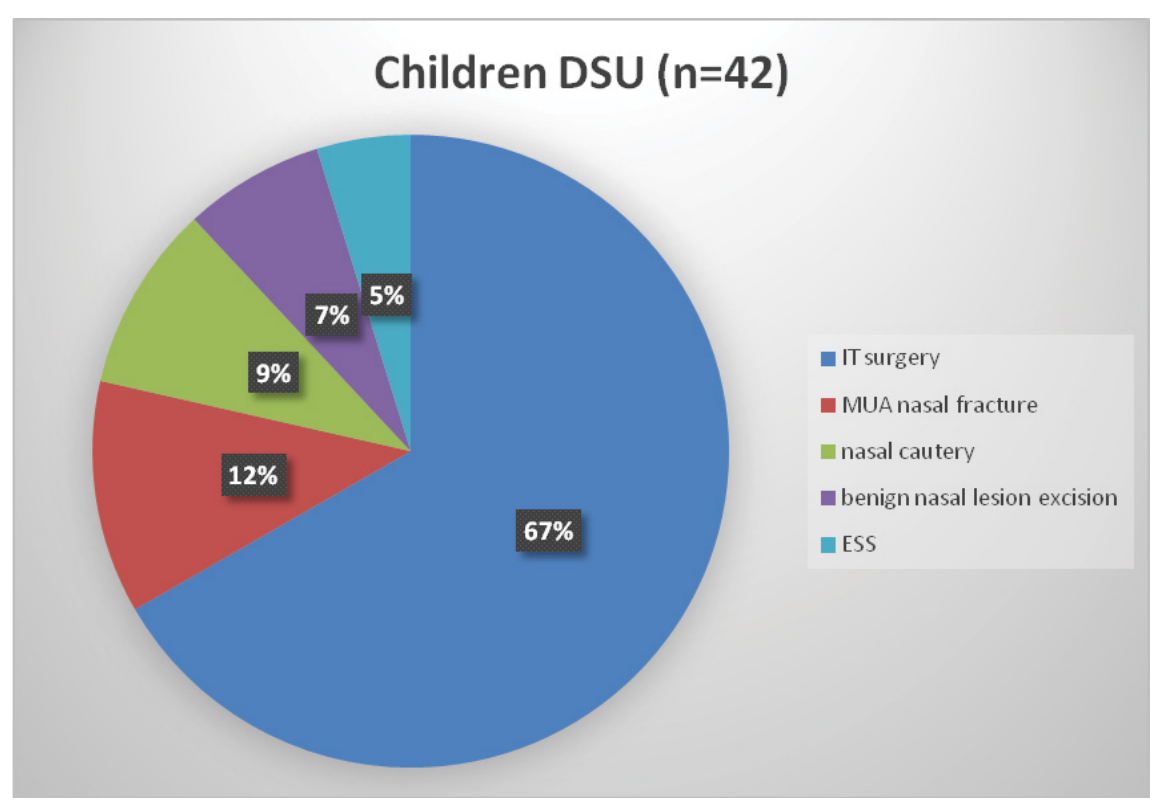

Figure 3: Sinonasal operations performed in children $(n=42)$ at DSU.

IT: Inferior Turbinate; MUA: Manipulation Under Anaesthesia; ESS: Endoscopic Sinus Surgery 
reduction and nasal cautery were the commonest operations performed in children; septoplasty, inferior turbinoplasty and sinus surgery were the commonest operations in adults (Figure 3).

Majority of patients (99\%) report an overnight post-op pain score of $<5$ on the visual analogue scale (VAS). Three patients (2.6\%) had a pain score $\geq 5$ at interview. Closed nasal fracture reduction and septorhinoplasty were associated with moderate pain (VAS 3-5) in first 24 hours post-op. One patient who reported a VAS pain score of 6 also had tonsillectomy along with his septo-turbinoplasty (Figure 4).
Apart from post-op pain, the most common complaint immediately post-op was nose bleeding (23\%). All 26 patients who reported this complication recorded the bleeding as minor and not needing any intervention. Four adult patients (5.6\%) reported mild dizziness following their general anaesthetic which settled down the following day. One patient $(0.9 \%)$ experienced post-operative nausea that settled with antiemetic medication. No delayed discharge or re-admission was reported during the study period (Figure 5).

The overall patients' experience and satisfaction score was $100 \%$ for rhinology day case surgery with excellent feedback

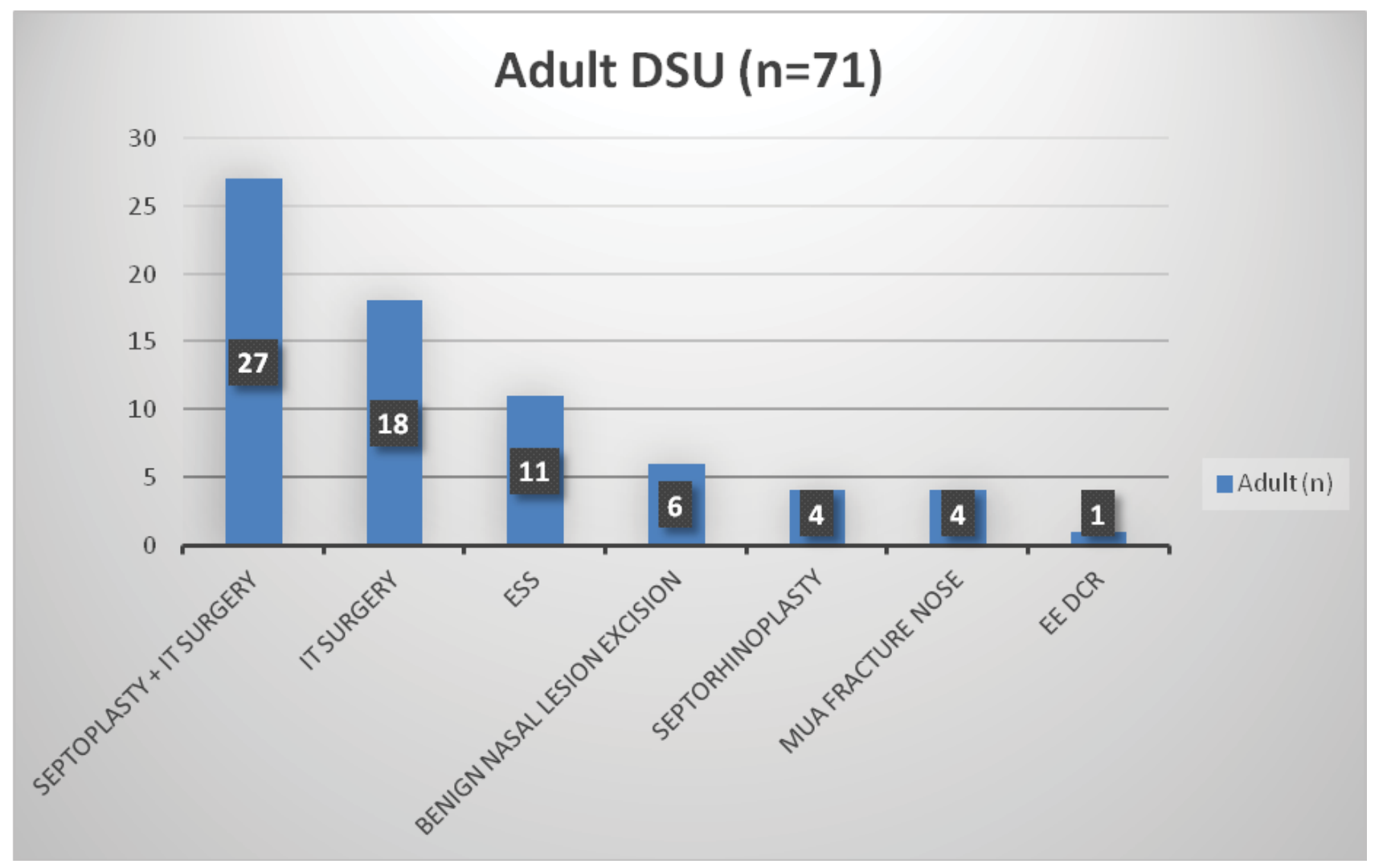

Figure 4: Sinonasal operations performed in adults $(n=71)$ at DSU.

IT: Inferior Turbinate; ESS: Endoscopic Sinus Surgery; MUA: Manipulation Under Anaesthesia; EE DCR: Endoscopic Endonasal Dacryocystorhinostomy

\begin{tabular}{|c|c|c|c|c|c|c|c|c|c|c|c|c|}
\hline \multirow[t]{3}{*}{ Pain overnight } & \begin{tabular}{|c|}
$\begin{array}{c}\text { present in } 25 \\
\text { patients }\end{array}$ \\
\end{tabular} & \multirow[b]{2}{*}{88} & \multirow[b]{2}{*}{12} & \multirow[b]{2}{*}{7} & \multirow[b]{2}{*}{4} & \multirow[b]{2}{*}{1} & \multirow[b]{2}{*}{1} & \multirow[b]{3}{*}{6} & \multirow[b]{3}{*}{7} & \multirow[b]{3}{*}{8} & \multirow[b]{3}{*}{9} & \multirow[b]{3}{*}{10} \\
\hline & Number of patients & & & & & & & & & & & \\
\hline & Pain scale & 0 & 1 & 2 & 3 & 4 & 5 & & & & & \\
\hline \multirow[t]{3}{*}{$\begin{array}{l}\text { Pain at the time } \\
\text { of interview }\end{array}$} & $\begin{array}{c}\text { present in } 29 \\
\text { patients }\end{array}$ & \multirow{2}{*}{84} & \multirow[b]{2}{*}{7} & \multirow[b]{2}{*}{12} & \multirow[b]{2}{*}{5} & \multirow[b]{2}{*}{2} & \multirow[b]{2}{*}{2} & \multirow[b]{2}{*}{1} & & & & \\
\hline & Number of patients & & & & & & & & & & & \\
\hline & Pain scale & 0 & 1 & 2 & 3 & 4 & 5 & 6 & 7 & 8 & 9 & 10 \\
\hline
\end{tabular}

Figure 5: Post-op pain score overnight and at day 1 following day case surgery. 
from patients. All patients reported that they would be happy to undergo a sinonasal surgery on a day case basis.

\section{Discussion}

This study represents a continuous evaluation of our day case rhinology surgical service focusing on patients' satisfaction using this service across all age groups.

Over the years, there has been considerable controversy with contradictory conclusions drawn regarding suitability of sinonasal surgery performed as day case surgery. Most of these studies assessed septoplasty. One of the earliest studies published on day case rhinology surgery is a multi-centre study in 1996 looking at septoplasty and concluded this could be safely performed as day-case surgery [8]. Philpott and Banerjee [9] had proposed $92 \%$ of septoplasty can be done as day case based on their audit [9]. Other rhinology day case surgery published apart from septoplasty includes work done by Danielsen [10] who described out-patient endoscopic ethmoidectomy without complications. Banfield, et al. [11] and Georgalas, et al. [12] published on day case rhinoplasty and septorhinoplasty and reported an admission rate of $15 \%$ and $3 \%$ respectively. The re-admission rates associated with day-case septoplasty, mainly due to bleeding, ranged from $2 \%$ [9], 5\% [13], 8.8\% [14] to $11.4 \%$ [15]. Georgalas, et al. [12] has reported a higher incidence of bleeding in patients prescribed diclofenac after septoplasty [14]. However, more work needs to be done and randomized controlled studies are needed in this area. In our series, $23 \%$ of patients reported to have minor nose bleed in the immediate post-op period of which all settled spontaneously with no intervention required. None of the patient had their discharge plan amended as a result of this. At telephone interview, there was no complaint of ongoing nose bleed and no re-admission was recorded due to post-op bleeding.

Several studies have shown that intensity of postoperative pain can be the decisive factor in patients' satisfaction [16-21]. In our cohort, 25 (22\%) patients reported post-op pain at discharge but none scored $>5$. Although this has risen to 29 patients (25\%) patients at interview, majority had a pain score of $<4$ with an exception of 1 patient (VAS $=6$ ) who had tonsillectomy and septoplasty simultaneously. The pain is thought to be attributed to his tonsillectomy rather than the septoplasty. This does correlates well with the high satisfaction level observed in our cohort. In younger children, the Wong-Baker FACES pain rating scale which is a validated pain scale, was used to record pain [22]. This has been shown to be effective in measuring pain severity in children $[23,24]$.

In our case series, only 1 patient reported post-operative nausea and vomiting (PONV). Four patients experienced short-lived dizziness after surgery which settled without any intervention. These factors had not impacted on patient overall satisfaction. Nevertheless, PONV has been reported to be a strong predictor of patient satisfaction $[17,18]$.

The overall patient satisfaction response obtained was good when compared with other studies, though, the data may be skewed by absence of data collection from patients who could not be contacted or acquiescence bias that may occur in telephone interviews.

There is published literature highlighting a number of factors such as waiting time for surgery, DSU facilities and design, cleanliness, professionalism of nursing and medical staffs, meeting the anesthesiologist pre-op, post-operative pain management, complications such as nose bleed, after effects of the general anaesthetic such as nausea and vomiting, and information provided post-operatively can impact on patient satisfaction. These factors need to be addressed and reviewed regularly when carrying out audit of any day case ENT surgical service.

There are several limitations to this study. First of all, only 113 of 160 patients were included due to inability to complete a telephone review, which could lead to the possibility of selection bias for the results. Secondly, post-op assessment of a young child can be difficult and inaccurate. Finally, the true cost assessment was not evaluated in this study.

\section{Conclusions}

Day case sinonasal surgery, in selected patients, is welltolerated by both children and adults. The overall patients' satisfaction in our centre is high with minimal reported postop complications none needing escalation of treatment or a more prolonged hospital stay.

\section{Conflict of Interest}

None declared by all authors.

\section{Ethical Approval}

This data collection is part of our ongoing service evaluation.

\section{References}

1. (1992) Guidelines for day case surgery, revised edition. Commission on the provision of surgical services. London: The Royal College of Surgeons of England.

2. Pézier T, Stimpson P, Kanegaonkar RG, et al. (2009) Ear, nose and throat day-case surgery at a district general hospital. Ann R Coll Surg Engl 91: 147-151.

3. Tysome JR, Padgham MD (2006) A comparative study of patient satisfaction with day-case and in-patient major ear surgery. J Laryngol Otol 120: 670-567.

4. Vowles R, Loney E, Williams $\mathrm{H}$, et al. (2000) Is paediatric daycase tonsillectomy desirable? The parent's perspective. Int J Clin Pract 54: 225-227.

5. Bartley JR, Connew AM (1994) Parental attitudes and postoperative problems related to paediatric day stay tonsillectomy. N Z Med J 107: 451-452.

6. Kanerva M, Tarkkila P, Pitkaranta A (2003) Day case tonsillectomy in children: parental attitudes and consultation rates. Int $J$ Paediatr Otorhinolaryngol 67: 777-784.

7. Otte DI (1996) Patients' perspectives and experiences of daycase surgery. J Adv Nurs 23: 1228-1237.

8. Benson-Mitchell R, Kenyon G, Gatland D (1996) Septoplasty as a day-case procedure - a two centre study. J Laryngol Otol 110: 129-131. 
9. Philpott CM, Banerjee AR (2005) Is there a role for more daycase septal surgery. J Laryngol Otol 109: 129-131.

10. Danielsen A (1992) Functional endoscopic sinus surgery on a day-case out-patient basis. Clin Otolaryngol Allied Sci 17: 473477.

11. Banfield GK, McKiernan D, Hinton AE (2000) Day case rhinoplasty. J R Army Med Corps 146: 212-214.

12. Georgalas C, Paun S, Zainal A, et al. (2002) Assessing day-case septorhinoplasty: Prospective audit using patient based indices. J Laryngol Otol 116: 707-710.

13. Hogg RP, Prior MJ, Johnson AP (1999) Admission rates, early readmission rates and patient acceptability of 142 cases of day case septoplasty. Clin Otolaryngol Allied Sci 24: 213-215.

14. Georgalas C, Obholzer R, Martinez-Devesa P, et al. (2006) Daycase septoplasty and unexpected re-admissions at a dedicated day-case unit: A 4-year audit. Ann R Coll Surg Engl 88: 202-206.

15. Srinivasan V, Arasaratnam RBS, Jankelowitz GA (1995) Day case septal surgery under general anesthesia and local anesthesia with sedation. J Laryngol Otol 109: 614-617.

16. Bain J, Kelly H, Snadden D, et al. (1999) Day surgery in Scotland: Patient satisfaction and outcomes. Qual Health Care 8: 86-89.
17. Jenkins K, Grady D, Wong J, et al. (2001) Post- operative recovery: Day surgery patient' preferences. Br J Anaesth 86: 272-274.

18. Myles PS, Williams DL, Hendrata M, et al. (2000) Patient satisfaction after anaesthesia and surgery: Results of a prospective survey of 10,811 patients. Br J Anaesth 84: 6-10.

19. Scott NB, Hodson M (1997) Public perceptions of post-operative pain and its relief. Anaesthesia 52: 438-442.

20. Beauregard L, Pomp A, Choiniere M (1998) Severity and impact of pain after day surgery. Can J Anaesth 45: 304-311.

21. Lemos $P$, Pinto $A$, Morais $G$, et al. (2009) Patient satisfaction following day surgery. J Clin Anesth 21: 200-205.

22. Garra G, Singer AJ, Taira BR, et al. (2010) Validation of the Wongbaker faces pain rating scale in pediatric emergency department patients. Acad Emerg Med 17: 50-54.

23. Franck LS, Greenberg CS, Stevens B (2000) Pain assessment in infants and children. Pediatr Clin North Am 47: 487-512.

24. McGrath PA (1990) Pain assessment in infants and children. In: PA Mcgrath, Pain in children: Nature, Assessment, and Treatment. New York, NY: The Guildford Press, 4-87. 